\title{
Hexagonal Boron Nitrides (White Graphene): A Promising Method for Cancer Drug Delivery
}

This article was published in the following Dove Press journal:

International Journal of Nanomedicine

\author{
Shazid Md Sharker \\ Department of Pharmaceutical Sciences, \\ North South University, Dhaka I229, \\ Bangladesh
}

\begin{abstract}
Advances in low-dimensions nanomaterials drug-carrier have rapidly translated into clinical practice. Interestingly, the two-dimensional (2D) nanomaterials of hexagonal boron nitride (h-BN), so-called "white graphite" are relatively less explored compared to the post popular 2D graphene oxide (GO). However, the unique properties of h-BN nanomaterials make them well suited for the delivery of chemotherapeutic in cancer treatment. Recent studies have shown that the h-BN is a potential candidate in biomedical sciences, both as nanocarriers and nano-transducers. In this review, we discuss the various physicochemical properties and important concepts involved in h-BN nanosheets as anticancer drug carriers. Keywords: h-BN, functionalization, biomedical potentiality, biomaterials, anticancer drug delivery
\end{abstract}

\section{Introduction}

Boron nitride $(\mathrm{BN})$ is a crystalline material composed of an equivalent stoichiometry of nitrogen $(\mathrm{N})$ and boron $(\mathrm{B})$ atoms. The $\mathrm{BN}$ material is available in different polymorphisms through cubic $\mathrm{BN}$ (c-BN), hexagonal $\mathrm{BN}$ (h-BN), wurtzite $\mathrm{BN}(\mathrm{w}-\mathrm{BN})$, and rhombohedral BN (r-BN) form, respectively. The h-BN and r-BN hold dense structure with sp2 hybridized B-N bonds, while w-BN and c-BN hold loose structure with sp3 hybridized bonds. Among them, c-BN (cubic) and h-BN (hexagonal) are the most studied structures. The c-BN is well-known as the second strongest material similar to the crystal lattice form of diamond, whereas (hexagonal) h-BN is a two dimensional (2D) layered structure. The h-BN is also referred to as white graphene and more similar to traditional graphene.

The building block of $\mathrm{h}-\mathrm{BN}$ is $\mathrm{B}$ and $\mathrm{N}$ atoms, which are assembled to form the honey comb like cage structure. In detail, the cage held together by the strong covalent bond in a hexagonal geometry shape. Also, the weak Vander Waals attraction forces are held the hexagonal interlayers (Figure 1). The B atom in this hexagonal interlayer typically placed precisely up or down the $\mathrm{N}$ atoms of the neighboring hexagonal layer. However, the distribution of electrons in $\mathrm{BN}$ is different from graphene's carbon-carbon $(\mathrm{C}-\mathrm{C})$ network structure. ${ }^{1}$ Notably, the electrons in the B-N bonds move towards electronegative $\mathrm{N}$ atoms resulting in the $\mathrm{B}$ atoms empty. In other word, it imparts a partial ionic property by polarizing the $\mathrm{BN}$ bond.

The h-BN shows different nano-architectures as nanotubes (BNNTs), nanowires, nano-fibers, nano-whiskers, nanocarpets, nano-flowers, etc. and the morphologies. Each of these nano-structures shows various prominent material features like high BET (Brunauer Emmett Teller)-surface area, bandgap, electrical insulation,
Correspondence: Shazid Md Sharker Department of Pharmaceutical Sciences, North South University, Plot \# 15, Block \# B, Bashundhara R/A, Dhaka 1229 Bangladesh

Tel +880-2-55668200, Ext: 628।

Email shazid.sharker@northsouth.edu 


\section{Boron + Partial positive charge \\ Nitrogen - Partial negative charge}

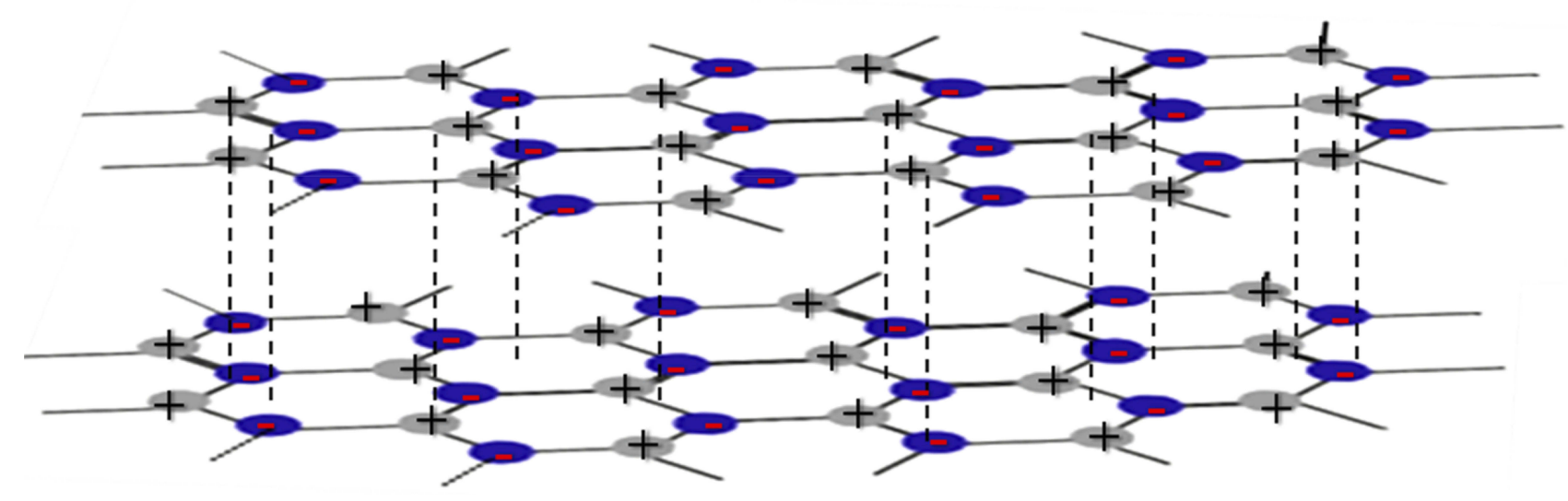

Figure I Structural model of h-BN nanosheets and Vander Waals attraction force between adjacent layers. The models of partial positive and negative charge on each boron and nitrogen atom.

thermal conductivity, etc. The multipurpose features of h-BN have drawn tremendous attention to use in material sciences, particularly in the drug delivery system (DDS). Additionally, the functions can be amplified either by applying new synthesis paths or by surface functionalization.

Hence, the current review focalizes on the recent success of h-BN nanomaterials with particular emphasis on the designing of drug delivery carriers and tuning nanostructure through functionalizing route. The review article also expresses the different functionalization routes for increasing the drug efficacy advantageous the trigger target delivery and biological uses for the therapeutic purpose. Additionally, the authors strongly think that the current review will assist in grasping the elaborations related to h-BN based DDS and their ongoing developments. ${ }^{1}$

\section{Biocompatibility and Bio-Applications}

By all means, there has been increasing potential interest in the biological use of h-BN nanomaterials for medical purposes, specifically drug carriers, biosensors, and tissue engineering. For this reason, the expected therapeutic applications of h-BN are required to study their biocompatibility and toxicity. ${ }^{2}$

The size, shape, form, and reactive surface of h-BN nanosheet determine biocompatibility. The study of micrometer-sized BN nanosheets proves in vitro biocompatible against Osteoblast-like cells. However, the nanosheets with a size less than $1 \mu \mathrm{m}$ and a depth below $100 \mathrm{~nm}$ were not so much biocompatible. It might be due to the boron radicals generate from the surface of the nanomaterial or, edges of nanosheet performed to generate ROS, leading to cell death. ${ }^{3}$

The in vivo biocompatibility studies on BN nanomaterials are still very limited. An introductory study of the pharmacokinetic behavior of the material proved proper in vivo distribution, with the first elimination rate from the systemic circulation. ${ }^{4}$ Moreover, BN nanosheets did not show to be cytotoxic when in vivo analysis on the planarians model, while any coating may decrease the possible cytotoxicity of the nanomaterial. ${ }^{5}$ Additional studies are requiring as the in vivo investigations guided to measure long-term biocompatibility in the case of chronic and acute effects.

\section{Functionalization}

The structural analog of graphene is h-BN, which can effectively be changed by different chemical conjugation reactions for functional materials. Nevertheless, the chemical inertness and stability of h-BN limit the require conjugation reaction and subsequently impeding versatile application. As a result, the modification in between surface and interface of h-BN may direct to overcome the existing limitation of functionalization with improved aqueous dispersibility and biocompatibility.

Chemically, the bonding electron cloud in between $\mathrm{B}$ and $\mathrm{N}$ atom in the h-BN stay more in $\mathrm{N}$ atom and less 
in $\mathrm{B}$ atom. The resulting imbalance of $\mathrm{BN}$ bonding electron or partial electro-negativity and electro-positivity generates a dipole interaction (Figure 1). Thus, any nucleophilic group can target the B atom, and the electrophilic group binds the $\mathrm{N}$ atomic site for chemical conjugation with the $\mathrm{BN}$ bond in the h-BN structure. Consequently, the covalent conjugation can be a preferable route for the functionalization of h-BN through the reactive radical species and the vacant $\mathrm{p}$ orbitals in B.

The classical functional groups like hydroxyl (-OH), amino $\left(-\mathrm{NH}_{2}\right)$, etc. have been attached on the surface of the h-BN structure. The treatments of acid, $\mathrm{O}_{2}$ plasma, and base results from the chemical interaction and binding of $\mathrm{OH}$ group with $\mathrm{B}$ (electron-less) sites of $\mathrm{BN}$ bonds. Moreover, this type of electrophilic interaction can increase by electronegative atoms doping. In the same way, ultrasonic etching with ammonia solution leads to attach $-\mathrm{NH}_{2}$ groups to the h-BN surface. Furthermore, the $-\mathrm{OH}$ group-containing h-BN can change to $-\mathrm{NH}_{2}$ group via heating and silane conjugating agents. All of these attempts allow the synthesis of newer functionalized h-BN nanomaterials. ${ }^{6}$

The oxidation reaction, which is favorable for graphene-based materials through piranha solution and hot Aqua Regia, is not convenient for h-BN based materials owing to its hydrophobic nature. In this case, the simultaneous thermolysis and hydrolysis via potent oxidizing agents can lead to the attachment of peroxide groups in the h-BN structure.

Furthermore, the typical pi-pi stacking, which is promptly used in graphene-like material, also helps in h-BN structure by mean of non-covalent modification. The structural defects remain unaffected from any incorporation in sp2 hybridized structures. In this way, different protein, polymer, and inorganic groups can be anchored to the surface of the h-BN structure. Thereby, the explored potentiality of h-BN for the loading of therapeutic agents can be explored by different functionalization reactions through the surface and interface of this graphene-like material (Figure 2).

Furthermore, the functionalization is a vital process to introduce new features and usage for a material. Fruitful physical and chemical methods can use as functionalization process of $\mathrm{h}-\mathrm{BN}$. Unique merits of functionalized h-BN materials, including aqueous solubility, biocompatibility, high processability have ensured diverse application in biomedical sciences and drug delivery systems. ${ }^{7}$

\section{Physical Functionalization}

The physical modification of $\mathrm{h}-\mathrm{BN}$ nanomaterials indicates the structural and morphological alterations. Commonly, the h-BN exhibits a laminar plate-like structure in its natural state. The thicknesses of the natural form of h-BN vary from dozens to hundreds of (nm) nanometers, and lateral size is more than $(\mu \mathrm{m})$ micrometers. In this way, their bulk structure can fabricate to zero-dimensional particle or cage, one-dimensional tube or ribbons or wire, two-dimensional sheets, and three-dimensional porous structures by different physical routes. This section will selectively introduce and discuss low-dimensional and porous structures of BN functional nanostructures. ${ }^{7}$

\section{Low-Dimensional BN Nanostructures}

Despite the new features and potentiality of BN nanomaterials, work on the fundamental physical aspects and advance uses of these nanomaterials has been minimal compares to well-explored carbon (C) nano-systems (For example, graphene oxide, carbon nanotubes, and others nano-carbon family). The difference might be imposed on the difficult work needed in the bulk-synthesis of low dimensional $\mathrm{BN}$ nanomaterials. Moreover, an h-BN and graphene combined hybrid materials would allow the introduction of advance structural features to be tailored.

However, the potential material synthesis methods (For example- chemical vapor deposition) can work for morphological fabrication and allow incorporating a new functional entity. The unique features are seemed to be explored in the advancement of $\mathrm{BN}$ structures. ${ }^{8}$

\section{Porous Structures}

The porous structures of $\mathrm{BN}$ are considered to be potential for drug delivery carriers. The porous form of $\mathrm{BN}$ could be obtained in hard/soft-templating or non-templating physical methods. Different templates, including activated carbon, silica, zeolites, graphene, and aerogels, can use for the preparation of porous BN. At the same time, nontemplate strategy could also employ in this attempt that is ensuring improves texture towards the porous form of BN nanostructures. ${ }^{7}$

\section{Chemical Functionalization}

The functionalization of the $\mathrm{BN}$ materials is comparatively very less explored, while graphite and h-BN both similar structural patterns. The functional modifications of carbonbased graphite have the potential advantage of many organic chemical reactions where it is analogous BN suffer 

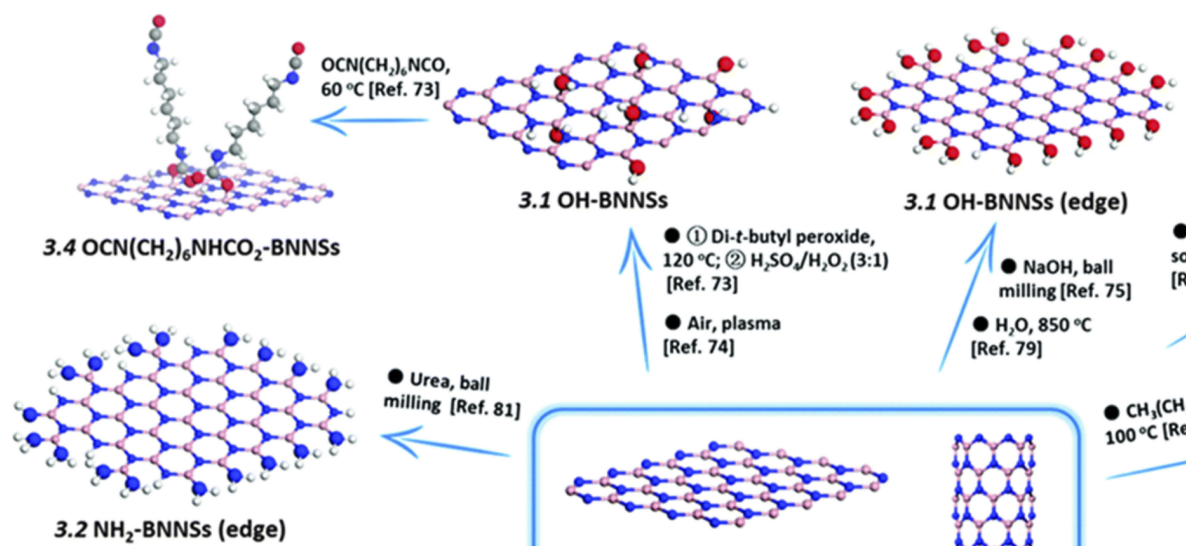

$3.2 \mathrm{NH}_{2}-\mathrm{BNNSs}$ (edge)

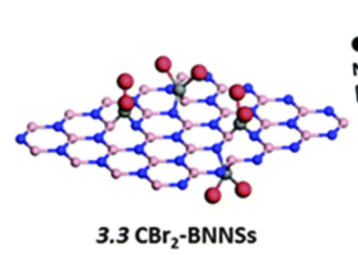

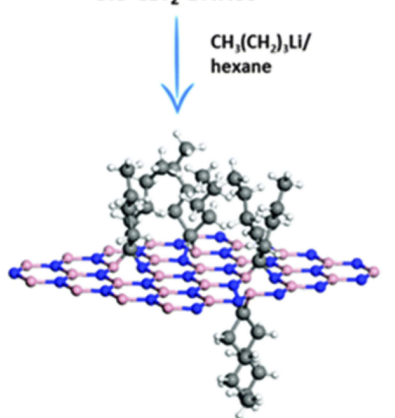

$3.3\left(n-\mathrm{C}_{4} \mathrm{H}_{9}\right)_{2} \mathrm{C}-\mathrm{BNNSS}$
- $\mathrm{CHBr}_{2}$ benzene, [Ref. 86]
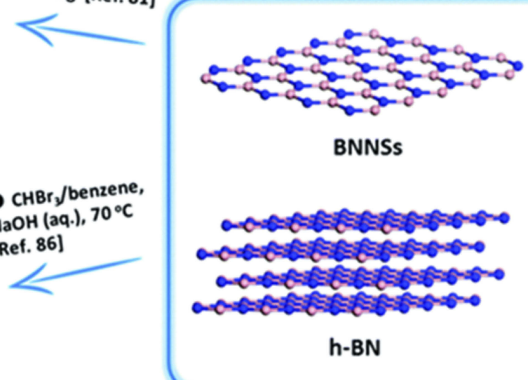

BNNSs
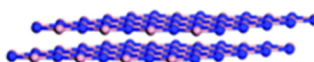

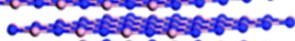

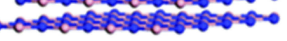

h-BN

$-(1) \mathrm{CH}_{3}\left(\mathrm{CH}_{2}\right)_{12} \mathrm{NH}_{2}$,
$60^{\circ} 180^{\circ} \mathrm{C}, \mathrm{N}_{2}$ flow;
(2) THF, sonication
[Ref. 85]

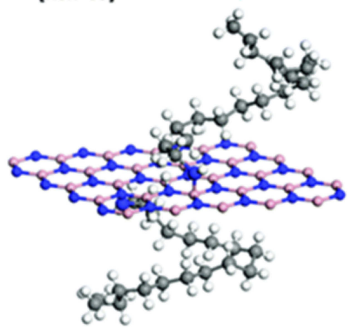

$3.2 \mathrm{CH}_{3}\left(\mathrm{CH}_{2}\right)_{17} \mathrm{NH}-\mathrm{BNNSS}$

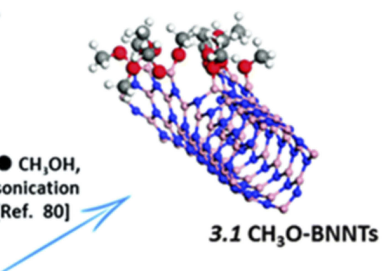

$\bullet \mathrm{H}_{2} \mathrm{O}, 850^{\circ} \mathrm{C}$
[Ref. 79]

3.1 $\mathrm{CH}_{3} \mathrm{O}-\mathrm{BNNTS}$

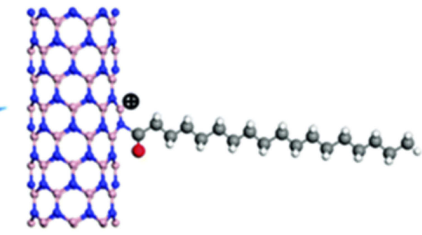

$3.4 \mathrm{CH}_{3}\left(\mathrm{CH}_{2}\right)_{16} \mathrm{CO}-\mathrm{BNNTS}$
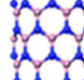

As

(1) Na." (naphthalene)/THF;

ANA

BNNTs

(2)1-Bromohexane [Ref. 87]
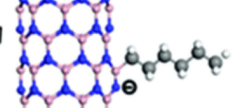

A

And

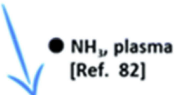

s.A.

3.3 $\mathrm{CH}_{3}\left(\mathrm{CH}_{2}\right)_{5}-\mathrm{BNNTS}$

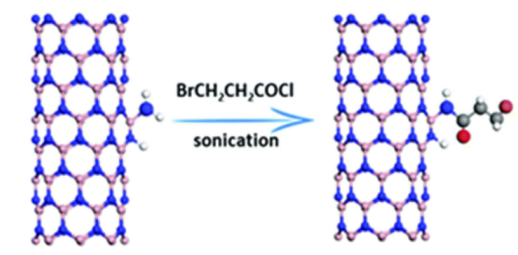

$3.2 \mathrm{NH}_{2}-\mathrm{BNNTS}$

$3.2 \mathrm{BrCH}_{2} \mathrm{CH}_{2} \mathrm{CONH}$-BNNTS

Figure 2 Schematics representation of different functionalization strategy of BN nanomaterials.

Notes: Weng Q, Wang X, Wang X, Bando Y, Golberg D. Functionalized hexagonal boron nitride nanomaterials: emerging properties and applications. Chem Soc Rev. 2016;45(14):3989-40I2. Published by The Royal Society of Chemistry. ${ }^{6}$

in common organic reaction for chemical functionalization. For this reason, different methods are continuously developed to overcome the existing limitations (Figure 2).

Interestingly, the polarization opening and equal conjugated pi bond are work on the basal planes of both graphite and h-BN for the typical chemical reaction. As a consequence, the new generated bonds will be present at even number position. Any functional group bonded with boron or nitrogen in $\mathrm{BN}$ nanostructure is bind in the $\mathrm{B}$ or $\mathrm{N}$ atom to balance the total charge. Moreover, the functional group can also be attached by pi-pi stacking on the $\mathrm{BN}$ surface. In the neighboring B-N unit, the BN structure can hold two groups, which are the simplest functionalization model. Both the theoretical and experimental studies revealed that bonded functional groups are not limited to neighboring locations while the charge and aromaticity are satisfied. Such a model could also implicate the respective h-BN functionalization process. Therefore, the different functional groups likes, hydroxyl $(-\mathrm{OH})$, amino $\left(-\mathrm{NH}_{2}\right)$, ether (-OR), amine (-NHR), acyl (-COR), alkyl (-R), and halogen) groups, as well as heteroatom ( $\mathrm{C}$ and $\mathrm{O})$, have been experimentally introduced into $\mathrm{BN}$ skeletons through chemical functionalization.

\section{Functional Properties and Biomedical Applications \\ Water Solubility}

$\mathrm{BN}$ nanostructures propelled significant interests in biomedical applications, such as tumor labeling, sensing, and trigger target nanomedicine delivery, etc. However, two important aspects have to address to develop the functionalized h-BN nanomaterials for biomedical applications.

First, the water solubility and homogeneity are the main concerns for any materials to use in the biological system. Bulk unmodified $\mathrm{BN}$ nanomaterials have 
significant hydrophobicity, which remains the challenge to introduce in physiologic environments. In this concern, surface functionalization and conjugation with the guest molecule or wrapping process become a successful effort to improve aqueous solubility and dispersibility of BN nanomaterials. Recently, satisfactory signs of progress have been made to overcome the existing limitation. For example, the highly water-soluble h-BN could be stabilized in the water at a concentration as high as $2 \mathrm{mg} / \mathrm{mL}^{8}$ As compared with other references, the achieved amount was two times higher and related to graphene oxide study reports. The achieved BN colloidal solution was found to stable more than a weak. Furthermore, the amine-functionalized (BN-NH2) that was developed ball milling method has been claimed to stable up to $30 \mathrm{mg} / \mathrm{mL}$ in the aqueous medium. In this way, chemical functionalization has been proved to be more effective in stabilizing the colloidal solution of BN nanomaterials. ${ }^{9,10}$

The biocompatibility is another main aspect for any materials to use in biologically. The biocompatibility of carbon-based nanomaterials depends on aspect ratio, concentration, purity, and the tissue or organ where it affected. Also, there are many toxicological investigations that have done that they shown BN nanomaterials are nontoxic or have very low toxicity. Assaying the $\mathrm{BN}$ nanomaterials with different purities and geometry, different dispersion reagents and cell types have correspondingly proved the biocompatibility. ${ }^{6}$

\section{Thermoresponsive Hydrogel Composite}

The fabrication of intrinsically hydrophobic bulk h-BN into hydrophilic (hydroxylated) $\mathrm{OH}-\mathrm{BN}$ nanomaterials facilitated the development of hydrogel composite. The sonication-based exfoliation exhibited $\mathrm{OH}-\mathrm{BN}$, which has enlarge and a couple-layered structure rather than the small fragments (Figure 3). The hydroxyl groups $(-\mathrm{OH})$ are found mainly on the ends of the nanosheets instead of on the basal surface. In one study, The PNIPAM/OH-BN hydrogel hybrid demonstrates a much quicker thermal performance contrast to the bare hydrogel, with an increase of $41 \%$ in thermal conductivity by including just 0.07 percent of $\mathrm{OH}-\mathrm{BN}$. The hydrogel formulation also exhibits stronger physical transition when heating, with the difference showing that $\mathrm{OH}-\mathrm{BN}$ has transferred on to the PNIPAM hydrogel to its superior thermal properties. Most notably, the dye-release test shows the potentiality to deliver drugs candidates. ${ }^{11}$

\section{Bio-Conjugated BN Nanomaterials}

The biomolecules including proteins, peptides, singlestranded DNA (ss-DNA), and carbohydrates have been conjugated onto BNNTs and, or h-BN nanosheets. The bio-conjugation facilitated water solubility and enhancement the biocompatibilities and bio-recognition capability. For example, the ferritin protein can physically adsorb onto BNNTs via natural ferritin-BNNT affinity or pyrene mediated pi-pi stacking, which is similar to the noncovalent functionalization of carbon nanotubes with biospecies. Moreover, the cytochrome-c, streptavidin, and glucose oxide could be analogously anchored with BNNTs by the same experimental procedure. Later, a peptide with several aromatic moieties (indole from tryptophan) was immobilized onto BNNTs, adapting the weak hydrophobic and pi stacking interactions between peptide units and BNNT

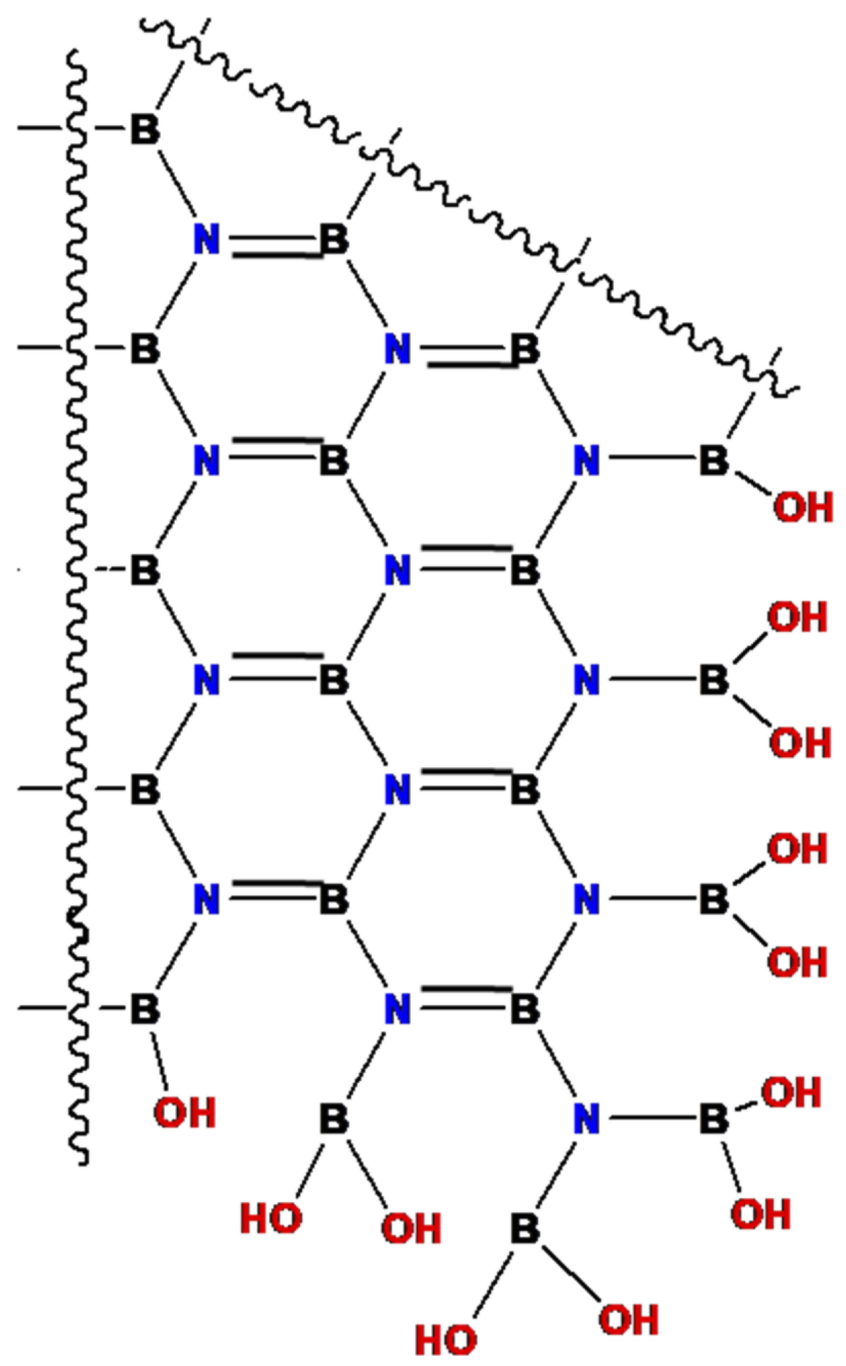

Figure 3 Fabricated hydrophilic (hydroxylated) $\mathrm{OH}-\mathrm{BN}$ nanomaterials for thermoresponsive hydrogel composite. 
surface. Following a conceptually similar strategy, ssDNA could wrap on BNNTs and lead to a homogenous conjugate solution, which allowed further fabrication or patterned BNNT structures. The same platform was utilized to conjugate ferritin proteins on h-BN nanosheets in aqueous media. Despite the differences in lateral sizes and thicknesses, all sheet surfaces were heavily covered with the protein molecules, and the resultant conjugates remained stable in aqueous dispersion after prolonged standing. ${ }^{6}$

\section{Hybrid h-BN with Gold Nanomaterials}

The hexagonal boron nitride (h-BN) with gold nanoparticles is considered as an attractive material for cancer drug delivery and photodynamic therapy (PDT) ${ }^{12}$ In one study, the h-BN was exfoliated by using chemical and physical methods, and later it was functionalized with gold $(\mathrm{Au})$ nanoparticles to obtain nano-hybrid (h-BN with $\mathrm{Au}$ ) particles. The h-BN-Au particles demonstrated inhibition of the proliferative activity of the MCF-7 cancer cell line in comparison with normal L929 cell lines after the 72-h incubation period. The investigational results make a new insight to consider the hybrid materials for use in anticancer therapy, biosensor design, tissue engineering, or in drug carriers.

\section{Boron as Biomaterials}

Boron can use in the coating of inert biomaterials such as metals and their alloy. These biomaterials find applications in a vast range of biomedical fields, such as surgical implants (joints, limbs, total hips, knees, artificial arteries, etc.), pacemaker leads, and cardiovascular nets. The boron-based Diamond-Like Carbon (DLC) coatings have been used for titanium alloys or stainless steel implants to avoid unwanted surface interactions with blood and tissues. DLC coatings show excellent hemocompatibility and tribological properties. For instance, they can act as a solid lubricant by forming a thin layer at the interface between articulation and attached components. However, DLC coating generally possesses poor adhesive properties toward biomedical metals and alloys such as titanium and stainless steel. ${ }^{13}$

Moreover, the boron compounds are increasingly recognized as preventative and chemotherapeutic agents. However, systemic administration of soluble boron compounds is hampered by their short half-life and low effectiveness. The hollow boron nitride (BN) spheres with controlled crystallinity had shown boron release, which decreases cell viability and increases prostate cancer cell apoptosis. In vivo experiments on subcutaneous tumor mouse models treated with BN spheres demonstrated significant suppression of tumor growth. The work indicates that hollow BN spheres may function as a new agent for prostate cancer treatment. ${ }^{14}$

\section{Anticancer Drug Delivery}

The nanocarrier encapsulated anticancer drug has the potential to maximized therapeutic effect and minimized unwanted side effects. However, it is still challenging to design such capable nanocarriers that are simultaneously biocompatible, soluble, and stable in physiologic environments, and could deliver required anticancer drugs. In this case, 2D h-BN like nanomaterials has great potential as anticancer nano-carriers (Table 1).

The hexagonal boron nitrides (h-BN) and boron nitride nanotubes (BNNT) are promising nanomaterials since they possess large surface area, excellent mechanical strength, and required biocompatibility. In one study, the potential use of BNNTs and h-BN as nanocarriers was comparatively investigated for use to deliver anticancer drugs (Figure 4). The observed data suggest that BNNT has a higher loading ability than h-BN nanosheets. Furthermore, the folic acid (FA) and hyaluronic acid (HA) conjugated delivery system have confirmed sufficient higher cellular uptake, which allows the death of increases number of cancer cells. ${ }^{15,16}$ In another experiment, the FA was grafted onto BN nanosheets (NS) via esterification reaction, and doxorubicin hydrochloride (DOX) was loaded onto BNNS-FA complexes. The BNNS-FA/DOX complexes have found stable at $\mathrm{pH} 7.4$ but effectively released DOX at $\mathrm{pH}$ 5.0, which exhibited a $\mathrm{pH}$-sensitive and sustained release pattern. Furthermore, the BNNS-FA/DOX complexes could be recognized and internalized explicitly by HeLa cells via FA receptormediated endocytosis. ${ }^{17}$

Also, the thermal substitution methods to fabricate entirely water-soluble and porous boron nitride (BN) materials have shown unprecedentedly high degrees of hydroxylation $(\mathrm{OH})$. These hydroxylated $\mathrm{BNs}(\mathrm{OH}-\mathrm{BN})$ are biocompatible and can effectively load anticancer drugs up to contents three times exceeding their weight. Therefore compare with free drugs, the drug-loaded BN carriers have shown a much higher anticancer effect against LNCaP cancer cells. ${ }^{18,19}$ Similarly, the hydrophilicity and drug loading capacity of mesoporous $\mathrm{SiO} 2$ coated BN nanomaterials have found adsorb more DOX drugs 
Table I Recent Studies of h-BN for Advanced Drug Delivery System

\begin{tabular}{|c|c|c|c|c|}
\hline No. & Form of Boron Nitride (BN) & Loading/Modifications & Application & References \\
\hline I. & h-BN & $\begin{array}{l}\text { I. Doxorubicin } \\
\text { 2. Carboplatin } \\
\text { 3. Cisplatin } \\
\text { 4. Hydroxylation } \\
\text { 5. CPG oligodeoxynucleotides } \\
\text { 6. Pd and copper (II) phthalocyanine }\end{array}$ & $\begin{array}{l}\text { Anticancer therapy } \\
\text { Anticancer therapy } \\
\text { Anticancer therapy } \\
\text { Hydrogel composite } \\
\text { Gene therapy } \\
\text { Phototherapy }\end{array}$ & $\begin{array}{l}{[14,16,27]} \\
{[30]} \\
{[32,34]} \\
{[10]} \\
{[22,23]} \\
{[11,19]}\end{array}$ \\
\hline 2. & $\mathrm{~B}_{24} \mathrm{~N}_{24}$ & I. 5-Fluoro uracil & Anticancer therapy & [32] \\
\hline 3. & $\mathrm{~B}_{12} \mathrm{~N}_{12}$ & I. Cisplatin & Anticancer therapy & [33] \\
\hline 4. & Hollow spheres & I. DLC & Biomaterials & {$[12,13]$} \\
\hline 5. & Nanotube (BNNT) & $\begin{array}{l}\text { I. } \mathrm{B}(10) \text { isotope } \\
\text { 2. Carboplatin } \\
\text { 3. Curcumin }\end{array}$ & $\begin{array}{l}\text { Radiation therapy } \\
\text { Anticancer therapy } \\
\text { Anti-inflammatory }\end{array}$ & $\begin{array}{l}{[28,29]} \\
{[30]} \\
{[21]}\end{array}$ \\
\hline
\end{tabular}

than the bare BN nanomaterials. The loaded BN nanomaterials have shown an improved cancer cell killing ability. These attempts inspired fabricated of BN nanomaterials coated with europium doped sodium gadolinium fluoride (NaGdF4-Eu) for drug delivery. Moreover, the NaGdF4$\mathrm{Eu}$ found to be advantageous for target directing and manipulating in an external magnetic field. Furthermore, the Boron nitride nanotubes@NaGdF4:Eu composites having core@shell structures show a significantly higher cellular uptake and chemotherapy deliverable in the presence of an external magnetic field than that in its absence. ${ }^{20}$

At the same time, the BNNT@mesoporous silica hydrides for controllable surface zeta potential materials have shown higher suspension ability that facilitated intracellular endocytosis of doxorubicin. ${ }^{21}$ Moreover, the BNNTs encapsulated drug (curcumin) has found to efficient cellular uptake ability and in vitro release potentiality, which associated predictable antiinflammatory effects of curcumin. ${ }^{22}$ Another study provided new insight towards the promising application of adenine-functionalized macromer (A-PPG) with the h-BN system in controlled release drug delivery systems. The water-soluble A-PPG directly self-assembles into a longperiod stacking-ordered lamellar structure over the surface of hexagonal boron nitride (h-BN) in aqueous solution. This is because of, the efficient non-covalent interactions between A-PPG and BN nanosheets. The layer number of h-BN nanosheets can be easily tuned by altering the mass ratio of the A-PPG and h-BN blend, and the resulting exfoliated nanosheets. The h-BN nanosheet has also exhibited excellent temperature/pH-responsive behavior, biocompatibility, and extremely high drug-loading capacity. The observed features are highly desirable in traditional 2D nanomaterials. The newly functionalized 2D

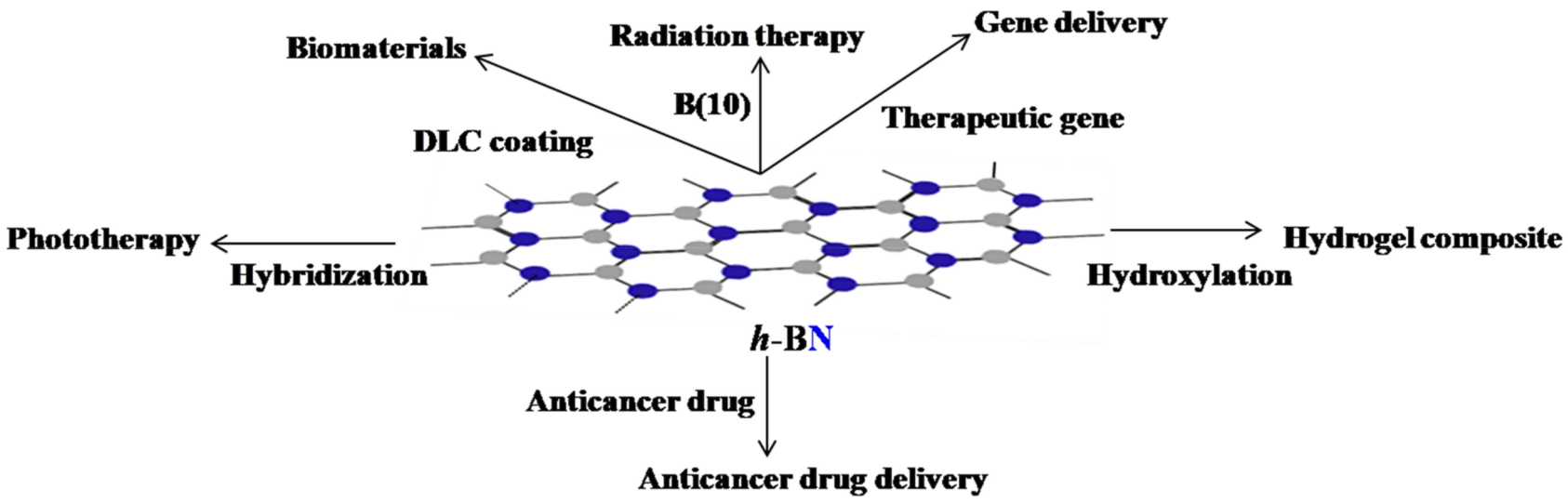

Figure 4 Schematic description of h-BN based different delivery system and their potential biological applications. 
nanocarrier shows the rapid release of drug in acidic $\mathrm{pH}$ and raising temperature condition. As a result, under particular cellular micro-environment, the $\mathrm{pH}$ and temperature-responsive carrier system could work like a safe multi-responsive 2D nanocarrier. ${ }^{23}$

\section{Gene Delivery}

The functionalized BN nanomaterials could be considered as a promising candidate to deliver therapeutic agents like- proteins, genes, and other biomolecules (Figure 4). In gene delivery, the therapeutic efficacy is mostly limited due to nuclease degradation and reduced cellular internalization. For example, the $\mathrm{CpG}$ oligodeoxynucleotides (CpG ODNs) hold a promising immunostimulatory activity in cancer immunotherapy (Table 1). In one study, boron nitride nanospheres (BNNS) have shown advantage carriers for $\mathrm{CpG}$ ODNs. The amino group grafted of mesoporous silica (MS)-functionalized BNNS appeared as a novel nanovector for $\mathrm{CpG}$ ODN delivery. Furthermore, the modification of BNNS with MS significantly improved the dispersity of BNNS and $\mathrm{CpG}$ ODN loading. In another study, the BNNS@MS-NH2 exhibited no cytotoxicity and enhanced the delivery of $\mathrm{CpG}$ ODNs into macrophages. The BNNS@MS-NH2/CpG ODN complexes triggered enhanced immunostimulation and induced higher amounts of cytokines. These findings provide a promising strategy for enhancing $\mathrm{CpG}$ ODNmediated cancer immunotherapy. ${ }^{24}$ Also, the positively charged BNNS could develop by coating with chitosan preparations. This strategy can use as carriers for the delivery of $\mathrm{CpG}$ oligodeoxynucleotides. The chitosancoated BNNS (BNNS-CS) have shown positive (+ ve) potential and improved water solubility and dispersibility. At the same time, loading capacity and cellular uptake ability of $\mathrm{CpG}$ oligodeoxynucleotides have significantly increased owing to surface +ve charge. Therefore the $\mathrm{CpG}$ oligodeoxynucleotides loaded BNNS-CS is potential to increase interleukin-6 production and tumor necrosis factor- $\alpha$ production compare to standard Lipofectamine ${ }^{\mathrm{TM}} 2000$ and bare BNNS carriers. $^{25}$

\section{h-BN Based Therapeutic Agent Delivery}

The in situ deposition of $\mathrm{Pd}$ nanoparticles on the surface of the h-BN nanosheets ( $\mathrm{Pd} @ \mathrm{OH}-\mathrm{BNNS})$ has endowed the photothermal property (Figure 4). Moreover, the $\mathrm{Pd} @ \mathrm{OH}-$ BNNS as a drug delivery carrier exhibited a high loading capacity for the anticancer doxorubicin drug. A decrease in $\mathrm{pH}$ triggered the release of doxorubicin from the nanohybrids, and increases in glutathione concentration and nearinfrared (NIR) irradiation. As a result, the tumor growth was remarkably inhibited in mice after two weeks of treatment. Additionally, the $\mathrm{Pd} @ \mathrm{OH}-\mathrm{BNNS} /$ doxorubicin injections have demonstrated the high efficacy of combined chemotherapy and photothermal therapy (PTT) (Table 1). ${ }^{26}$ Also, the introduction of magnetic nanoparticles allows target tumor areas, and the cancer cells could destroy by hyperthermia release from magnetohyperthermia. The biocompatibility and anticancer effects of magnetohyperthermia agents have been demonstrated in biological assays. ${ }^{27}$ In another successful study, the therapeutic platform composed of h-BN conjugated DNA oligonucleotide, and copper (II) phthalocyanine $(\mathrm{CuPc})$ have played double key roles in photodynamic therapy (PDT) as well as in situ monitoring and imaging of miR-21. The PDT efficacy and biocompatibility of such an integrated nanoplatform have been ascertained both in vitro and in vivo study. Moreover, the proposed nano-prove are also potential for diagnosis and guided photodynamic therapy. ${ }^{28}$

\section{BN Carrier for the Targeted Nano-Radio Therapeutic Agent}

The fabricated $\mathrm{BN}$ nanostructures materials have unique physical, chemical, optical, and mechanical features, which are potential features for complex biological systems. Currently, the boron neutron captures therapy (BNCT), a kind of radiotherapy draw attention for use against cancer cells. In this therapy, a thermal neutron beam focused on boron-10 isotope that generates a lithium atom, a gammaray, and an alpha particle, allowing the destruction of target tumor tissues. Therefore, boron neutron capture therapy (BNCT) has been extensively studied to use in different malignant tumors, like cerebral glioblastoma.

In one such work, the BNNTs used as a boron-10 carrier for BNCT and to demonstrate its potentiality (Figure 4). The results show that this nanomaterial is a promising candidate for cancer therapy through BNCT. $^{29}$ Furthermore, the boron nitride nanotubes functionalized with biocompatible poly-l-lysine and fluorescent (quantum dots) probe with folic acid, which simultaneously allows tracking and targeting the tumor. The investigational data have shown potential nanocarrier in the presence of glioblastoma tumors. ${ }^{30}$ 


\section{DDS Evaluated Molecular Dynamics}

\section{Simulations}

The delivery of the drug on-site should limit the side effects of the drug. The interactions of different anticancer drugs and h-BN nanosheet as a carrier system have been further calculated in Molecular Dynamic (MD) simulations and Density Functional Theory (DFT) (Table 1). The simulated calculation shows that the adsorption of drugs onto the h-BN carrier is spontaneous and exothermic. Furthermore, the orbital energy and density of state (DOS) measurements show decreasing the HOMO to the LUMO energy gap of h-BN nanosheet upon the adsorption of anticancer drugs. At the same time, the quantum molecular description indicates the increasing chemical reactivity of loaded h-BN nanocarriers. In this prospect, the energy decomposition analysis (EDA) shown that the dispersion interaction plays a dominant role in this type of loading process. Moreover, the interaction energy values calculated in MD indicated lower (absolute value) in acidic $\mathrm{pH}$ compare to neutral $\mathrm{pH}$. These observations can contribute to a $\mathrm{pH}$-responsive drug delivery system for the released of the anticancer drug within the target cancer cells. ${ }^{31}$ Also, the average interaction energy values in acidic conditions are lower than corresponding values obtained at neutral $\mathrm{pH}$, which indicated that the drug could be released into the target cancer cells.

In the density functional theory, the $\mathrm{BN}\left(\mathrm{B}_{24} \mathrm{~N}_{24}\right)$ nanostructures are explored as a drug delivery system for the 5-fluorouracil drug. This drug chooses to bind via its oxygen atoms to the $\mathrm{B}$ atoms. It was observed that the introduction of more negative $(-11.90 \mathrm{kcal} / \mathrm{mol})$ adsorption energy, the electronic properties of Al-doped $\mathrm{BN}$, are remarkably sensitive to the drug adsorption. Also, the proposed drug release mechanisms indicate that drug and $\mathrm{BN}$ cluster is greatly protonated, thereby releasing the drug from the surface of the BN cluster. ${ }^{32}$ Moreover, the reactivity and electronic sensitivity of the synthesized $\mathrm{BN}$ $\left(\mathrm{B}_{12} \mathrm{~N}_{12}\right)$ nanocluster to cisplatin $(\mathrm{CP})$ anticancer drug had shown favorable for adsorption. The conduction level of BN nanocluster meaningfully stabilizes, and the valence level shifts to higher energies. As a result, the HOMOLUMO energy gap significantly decreases. ${ }^{33}$

The molecular dynamics simulations study on BNNT revealed a substantial storage capacity. The numbers of confined molecules on the spatial organization allow quantifying the energy profiles and the ability of BNNT to carry anticancer drug-like carboplatin. The small radius of BNNT is presented as a large inner volume that allows convenient encapsulation of anticancer drug molecules. ${ }^{30}$ The comparative penetration rate of water molecules was also investigated between bulk $\mathrm{BN}$ and functionalized BNNT. It has been found that the diffusion rate was sufficiently higher in functionalized BNNT owing to water-based H-bond onto the available hydroxyl surface. In particular, this property allows higher drug loading by displacement of van der Waals interaction in BNNT inner surface. ${ }^{34}$

In Monte Carlo Simulation and Quantum Mechanical calculations, the encapsulation efficiency of cisplatin drug on single-walled carbon nanotube (CNT) and boron-nitride nanotube (BNNT) were evaluated on a comparative basis. The simulation data suggest that cisplatin at BNNT was more soluble species in water and appropriate for more encapsulation in aqueous solution. ${ }^{34}$

\section{Summary}

The field of nano-fabrication and functionalization is moving at a very rapid pace. The expected capability of a $2 \mathrm{D}$ type h-BN structure enables the researchers to generate new nanoscale materials. These advances have been brought together by the combination of different organic and inorganic building blocks on this 2D platform. In part, the explorations by expert material researchers have already confirmed the notable manipulation strategies of h-BN for various biomedical purposes. The new class h-BN (white graphene) holds an even more attractive platform than most studying graphene oxide (GO) to deliver the anticancer medications. In this way, the anticancer drug like doxorubicin or therapeutic gene can release at the target site. Thus, it is likely that anticancer drug-loaded h-BN will enter advances cancer therapy soon.

\section{Acknowledgements}

The author is thankful to the North South University, Department of Pharmaceutical Sciences, Bangladesh for supporting this work. The author is grateful for the Conference Travel and Research Grants (CTRG), North South University Grant (Grant No. CTRG-19/SHLS/02) for their support.

\section{Disclosure}

The author declares that there is no conflict of interest regarding the publication of this article. 


\section{References}

1. Mishra NS, Saravanan P. A review on the synergistic features of hexagonal boron nitride (white graphene) as adsorbent-photo active nanomaterial. Chem Select. 2018;3(28):8023-8034.

2. Lu F, Wang F, Cao L, Kong CY, Huang X. Hexagonal boron nitride nanomaterials: advances towards bioapplications. Nanosci Nanotechnol Lett. 2012;4(10):949-961. doi:10.1166/nnl.2012.1444

3. Mateti S, Wong CS, Liu Z, et al. Biocompatibility of boron nitride nanosheets. Nano Res. 2018;11(1):334-342. doi:10.1007/s12274017-1635-y

4. Ciofani G, Danti S, Nitti S, Mazzolai B, Mattoli V, Giorgi M. Biocompatibility of boron nitride nanotubes: an up-date of in vivo toxicological investigation. Int $J$ Pharm. 2013;444(1-2):85-88. doi:10.1016/j.ijpharm.2013.01.037

5. Salvetti A, Rossi L, Iacopetti P, et al. In vivo biocompatibility of boron nitride nanotubes: effects on stem cell biology and tissue regeneration in planarians. Nanomedicine. 2015;10(12):1911-1922. doi: $10.2217 / \mathrm{nnm} .15 .46$

6. Weng Q, Wang X, Wang X, Bando Y, Golberg D. Functionalized hexagonal boron nitride nanomaterials: emerging properties and applications. Chem Soc Rev. 2016;45(14):3989-4012. doi:10.1039/ C5CS00869G

7. Pakdel A, Zhi C, Bando Y, Golberg D. Low-dimensional boron nitride nanomaterials. Mater Today. 2012;15(6):256-265. doi:10.1016/S1369-7021(12)70116-5

8. Lin Y, Williams TV, Connell JW. Soluble, exfoliated hexagonal boron nitride nanosheets. $J$ Phys Chem Lett. 2009;1(1):277-283. doi: $10.1021 / \mathrm{jz} 9002108$

9. Xiao F, Naficy S, Casillas G, et al. Edge-hydroxylated boron nitride nanosheets as an effective additive to improve the thermal response of hydrogels. $A d v$ Mater. 2015;27(44):7196-7203. doi:10.1002/ adma.201502803

10. Zhi C, Bando Y, Tang C, Xie R, Sekiguchi T, Golberg D. Perfectly dissolved boron nitride nanotubes due to polymer wrapping. $J \mathrm{Am}$ Chem Soc. 2005;127(46):15996-15997. doi:10.1021/ja053917c

11. Ciani L, Ristori S. Boron as a platform for new drug design. Expert Opin Drug Discov. 2012;7(11):1017-1027. doi:10.1517/ 17460441.2012 .717530

12. Li X, Wang X, Zhang J, et al. Hollow boron nitride nanospheres as boron reservoir for prostate cancer treatment. Nat Commun. 2017;8:13936. doi:10.1038/ncomms 13936

13. Emanet M, Şen Ö, Çulha M. Evaluation of boron nitride nanotubes and hexagonal boron nitrides as nanocarriers for cancer drugs. Nanomedicine. 2017;12(7):797-810. doi:10.2217/nnm-2016-0322

14. Feng S, Zhang H, Yan T, et al. Folate-conjugated boron nitride nanospheres for targeted delivery of anticancer drugs. Int J Nanomed. 2016;11:4573. doi:10.2147/IJN.S110689

15. Weng Q, Wang B, Wang X, et al. Highly water-soluble, porous, and biocompatible boron nitrides for anticancer drug delivery. ACS Nano. 2014;8(6):6123-6130. doi:10.1021/nn5014808

16. Sharker SM, Alam MA, Shill MC, Rahman GS, Reza HM. Functionalized hBN as targeted photothermal chemotherapy for complete eradication of cancer cells. Int J Pharm. 2017;534(1-2):206-212. doi:10.1016/j.ijpharm.2017.10.025

17. Sukhorukova IV, Zhitnyak IY, Kovalskii AM, et al. Boron nitride nanoparticles with a petal-like surface as anticancer drug-delivery systems. ACS Appl Mater Interfaces. 2015;7(31):17217-17225. doi:10.1021/acsami.5b04101

18. Li X, Hanagata N, Wang X, et al. Multimodal luminescent-magnetic boron nitride nanotubes@NaGdF 4: Eu structures for cancer therapy. Chem Commun. 2014;50(33):4371-4374. doi:10.1039/C4CC00990H

19. Li X, Zhi C, Hanagata N, Yamaguchi M, Bando Y, Golberg D. Boron nitride nanotubes functionalized with mesoporous silica for intracellular delivery of chemotherapy drugs. Chem Commun. 2013;49 (66):7337-7339. doi:10.1039/c3cc42743a
20. Niskanen J, Zhang I, Xue Y, Golberg D, Maysinger D, Winnik FM. Boron nitride nanotubes as vehicles for intracellular delivery of fluorescent drugs and probes. Nanomedicine. 2016;11(5):447-463. doi: $10.2217 / \mathrm{nnm} .15 .214$

21. Cheng CC, Muhabie AA, Huang SY, et al. Dual stimuli-responsive supramolecular boron nitride with tunable physical properties for controlled drug delivery. Nanoscale. 2019.

22. Zhang H, Cheng T, Lai L, et al. BN nanospheres functionalized with mesoporous silica for enhancing CpG oligodeoxynucleotide-mediated cancer immunotherapy. Nanoscale. 2018;10(30):14516-14524. doi:10.1039/ C8NR03820A

23. Zhang H, Chen S, Zhi C, Yamazaki T, Hanagata N. Chitosan-coated boron nitride nanospheres enhance delivery of $\mathrm{CpG}$ oligodeoxynucleotides and induction of cytokines. Int J Nanomed. 2013;8:1783. doi:10.2147/IJN.S37465

24. Zhang Y, Guo R, Wang D, Sun X, Xu Z. Pd nanoparticle-decorated hydroxy boron nitride nanosheets as a novel drug carrier for chemo-photothermal therapy. Colloids Surf, B. 2019;176:300-308. doi:10.1016/j.colsurfb.2019.01.015

25. Ferreira TH, Faria JA, Gonzalez IJ, et al. BNNT/Fe3O4 system as an efficient tool for magnetohyperthermia therapy. $J$ Nanosci Nanotechnol. 2018;18(10):6746-6755. doi:10.1166/jnn.2018.15514

26. Liu J, Zheng T, Tian Y. Functionalized h-BN nanosheets as a theranostic platform for SERS real-time monitoring of MicroRNA and photodynamic therapy. Angew Chem. 2019;131(23):7839-7843. doi:10.1002/ange.v131.23

27. Ferreira T, Miranda M, Rocha Z, Leal A, Gomes D, Sousa E. An assessment of the potential use of BNNTs for boron neutron capture therapy. Nanomaterials. 2017;7(4):82. doi:10.3390/nano7040082

28. Ciofani G, Raffa V, Menciassi A, Cuschieri A. Folate functionalized boron nitride nanotubes and their selective uptake by glioblastoma multiforme cells: implications for their use as boron carriers in clinical boron neutron capture therapy. Nanoscale Res Lett. 2009;4 (2):113. doi:10.1007/s11671-008-9210-9

29. El Khalifi M, Bentin J, Duverger E, Gharbi T, Boulahdour H, Picaud F. Encapsulation capacity and natural payload delivery of an anticancer drug from boron nitride nanotube. Phys Chem Chem Phys. 2016;18(36):24994-25001. doi:10.1039/C6CP01387B

30. Khatti Z, Hashemianzadeh SM. Boron nitride nanotube as a delivery system for platinum drugs: drug encapsulation and diffusion coefficient prediction. Eur J Pharm Sci. 2016;88:291-297. doi:10.1016/j. ejps.2016.04.011

31. Hazrati MK, Javanshir Z, Bagheri Z. B24N24 fullerene as a carrier for 5-fluorouracil anti-cancer drug delivery: DFT studies. $J \mathrm{Mol}$ Graphics Modell. 2017;77:17-24. doi:10.1016/j.jmgm.2017.08.003

32. Onsori S, Alipour E. A computational study on the cisplatin drug interaction with boron nitride nanocluster. J Mol Graphics Modell. 2018;79:223-229. doi:10.1016/j.jmgm.2017.12.007

33. Vatanparast M, Shariatinia Z. Hexagonal boron nitride nanosheet as novel drug delivery system for anticancer drugs: insights from DFT calculations and molecular dynamics simulations. J Mol Graphics Modell. 2019;89:50-59. doi:10.1016/j.jmgm.2019.02.012

34. Roosta S, Hashemianzadeh SM, Ketabi S. Encapsulation of cisplatin as an anti-cancer drug into boron-nitride and carbon nanotubes: molecular simulation and free energy calculation. Mater Sci Eng C. 2016;67:98-103. doi:10.1016/j.msec.2016.04.100

35. Lin Y, Williams TV, Xu TB, Cao W, Elsayed-Ali HE, Connell JW. Aqueous dispersions of few-layered and monolayered hexagonal boron nitride nanosheets from sonication-assisted hydrolysis: critical role of water. J Phys Chem C. 2011;115(6):2679-2685. doi:10.1021/ jp110985w 


\section{Publish your work in this journal}

The International Journal of Nanomedicine is an international, peerreviewed journal focusing on the application of nanotechnology in diagnostics, therapeutics, and drug delivery systems throughout the biomedical field. This journal is indexed on PubMed Central, MedLine, CAS, SciSearch ${ }^{\mathbb{B}}$, Current Contents ${ }^{\mathbb{B}} /$ Clinical Medicine, $^{2}$
Journal Citation Reports/Science Edition, EMBase, Scopus and the Elsevier Bibliographic databases. The manuscript management system is completely online and includes a very quick and fair peer-review system, which is all easy to use. Visit http://www.dovepress.com/ testimonials.php to read real quotes from published authors. 